\title{
COMPARATIVE STUDY OF IMPACT DAMAGE IN BASALT-CARBON HYBRID COMPOSITES USING INFRARED THERMOGRAPHY AND ULTRASONIC C-SCAN
}

\author{
H. Zhang ${ }^{1 *}$, S. Sfarra ${ }^{2,3}$, F. Sarasini ${ }^{4}$, C. Ibarra-Castanedo ${ }^{1}$, X. Maldague ${ }^{1 \dagger}$ \\ ${ }^{1}$ Department of Electrical and Computer Engineering, Computer Vision and Systems Laboratory, Laval University, \\ Quebec, QC, G1V 0A6, Canada \\ ${ }^{2}$ Department of Industrial and Information Engineering and Economics, Las.E.R. Laboratory, University of \\ L'Aquila, Roio Poggio, AQ I-67100, Italy \\ ${ }^{3}$ Tomsk Polytechnic University, 634028, Tomsk, Savinykh St., 7, Russia \\ ${ }^{4}$ Department of Chemical Engineering Materials Environment \& UDR INSTM, Sapienza University of Rome, Via \\ Eudossiana 18, Rome, I-00184, Italy \\ †Presenting Author: xavier.maldague@gel.ulaval.ca \\ *Corresponding Author: hai.zhang.1@ulaval.ca
}

\begin{abstract}
In this paper, infrared thermography (IRT) and ultrasonic C-scan (UT) were used to investigate basalt fiber reinforced polymer (BFRP), carbon fiber reinforced polymer (CFRP) and basalt-carbon fiber hybrid specimens subjected to impact loading. Of particular interest, two different hybrid structures including sandwich-like and intercalated stacking sequence were analyzed. Three different impact energies ( $5 \mathrm{~J}, 12.5 \mathrm{~J}$ and $25 \mathrm{~J}$ ) were applied for the evaluation of the impact damage level in the different samples. The inspection by thermographic techniques of this type of laminates is an open matter to be discussed with the scientific audience. The results from pulsed phase thermography (PPT), principal component thermography (PCT) and partial least squares thermography (PLST) applied on raw thermal data were compared to understand the advantages and disadvantages of the two hybrid structures via a comprehensive analysis.
\end{abstract}

KEYWORDS: Basalt fiber, Carbon fiber, Hybrid composite, Infrared thermography, Ultrasonic C-scan,

\section{INTRODUCTION}

The infrared thermography (IRT) technique is based on the recording of images and is gaining increasing attention in the recent years due to its fast inspection rate, contactless nature, spatial resolution and acquisition rate improvements of infrared cameras. In addition, the development of advanced image processing techniques plays an important role in its exponential increment. IRT can be used to assess and predict the structural integrity beneath the surface by measuring the distribution of infrared radiation and converting the measurements into a temperature scale. The inspection of composite materials damaged after impact loading is among the application fields in which the IRT technique has been applied in the past. Into the whole set of experimental setups, optical excitation thermography has been applied due to its ability to retrieve quantitative information concerning the defects. In addition, mechanical excitation thermography is also attracting increasing attention due to the powerful excitation approach.

Given the high specific strength and stiffness, carbon fiber reinforced polymer composites (CFRP) are often used in the aerospace industry. However, their toughness is considerably low, therefore the impact damage resistance is not very high. Impact damage is one of the most important threats for the aerospace industry. Impact loading due to unwanted objects may occur during the process of manufacturing, assembly, maintenance, and operation. Impact damage can greatly affect the residual mechanical properties of CFRP even if the damage is barely visible. Several solutions have been proposed in the past to enhance the impact damage resistance including the toughening of the matrix material. Another solution is represented by fiber hybridization (usually with high strain to failure fibers). In this regard, glass fiber is the best option mainly because it is inexpensive. Basalt fibers can be considered as an interesting alternative to glass fibers taking into account the mechanical properties and the environmental benefits. This type of fiber, obtained from basalt rocks, has been demonstrated to be effective for the reinforcement of polymeric matrices. 
An in-depth study centred on the thermographic diagnosis of post-impact damage in hybrid composites composed of basalt and carbon fibers has not been well documented yet in the open literature, while concerning basaltic materials, a couple of works will be taken into account in view of the discussion of the experimental results obtained. In this paper, IRT and ultrasonic C-scan (UT) were used to inspect basalt fiber reinforced polymer (BFRP), CFRP and basalt-carbon fiber hybrid specimens. Interestingly, two different hybrid structures including sandwich-like and intercalated (i.e., alternating sequence of basalt and carbon fabrics) were explored, although three different impact energies (5 J, $12.5 \mathrm{~J}$ and $25 \mathrm{~J}$ ) were used for the evaluation of the impact damage level. The advantages and disadvantages of the two structures were studied. Pulse phase thermography (PPT), partial least squares thermography (PLST) and principal component thermography (PCT) techniques were used together to process the raw thermographic data. Finally, a comprehensive and comparative analysis was conducted in view of future industrial applications.

\section{RESULT ANALYSIS}

UT (5 MHz) technique using the water immersion method was used in reflection mode and the back wall signals were recorded in order to obtain raster images, as shown in Fig. 1.

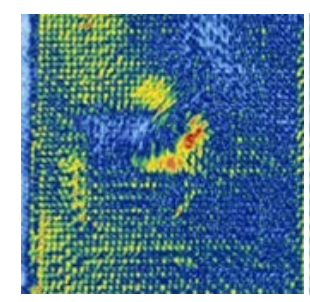

(a)

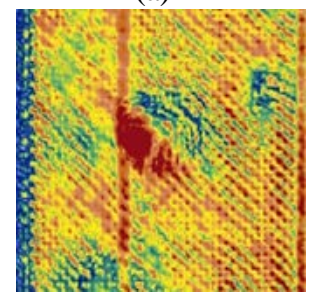

(e)

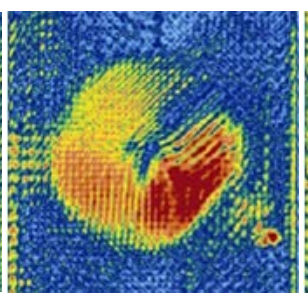

(b)

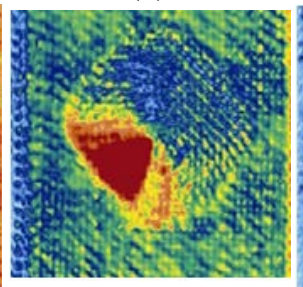

(f)

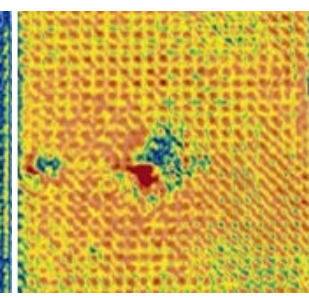

(c)

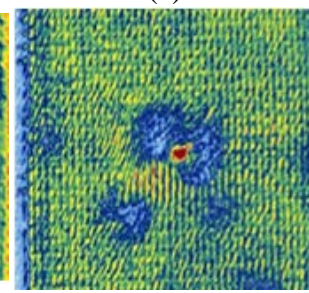

(g)

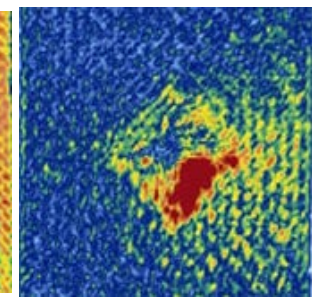

(d)

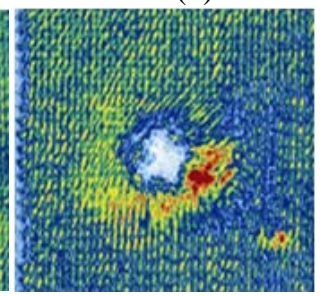

(h)

Figure 1: UT results: (a) B: 5 J, (b) B: 12.5 J, (c) C: 5 J, (d) C: 12.5 J, (e) BCs: 5 J, (f) BCs: 12.5 J, (g) BCa: 5 J, (h) BCa: 12.5 J.

BFRP specimens show larger delaminated areas than CFRP specimens (Figs. 1a - 1d). On one hand, the delaminated area caused by the impact at $12.5 \mathrm{~J}$ (Fig. 1b) is larger than that at $5 \mathrm{~J}$ (Fig. 1a). On the other hand, BCs shows clearer delaminated areas than BCa (Figs. 1e - 1h).

\section{CONCLUSIONS}

Absorbed energy Ea was more easily converted into delamination in BFRP thanks to its lower interlaminar shear strength. On the contrary, Ea created back surface splitting in CFRP samples due to their inherent limited ductility. Hybridization with basalt fibers improved the toughness of carbon-based laminates to different extent depending on the stacking sequence. The basaltcarbon fiber hybrid specimens (BCs) with sandwich-like structure show a pronounced delaminated area similar to the BFRP specimens, while the basalt-carbon fiber hybrid specimens (BCa) with alternately stacked structure show similar mechanical characteristics to those of CFRP. Summarizing, langer delaminated areas were detected in BCs specimens than in BCa specimens. This happened because the delaminations are probably more than in BCa specimens. However, they are less extended due to the multiple basalt-carbon interfaces. Finally, BCa shows bulge on the back side of the impacted zones. Interestingly, cross-shaped defects were detected in BCs specimens. This behavior can be attributed to the fact that the carbon fiber core plays an important role during the impact tests. The latter point of interest will be studied more in-depth in the future. 